\title{
PCHD-Based Passivity Control of VSC-HVDC Connected Large Wind Farm
}

\author{
Xinming Fan ${ }^{1}$, Lin Guan ${ }^{1}$, Chengjun Xia ${ }^{1}$, He Jianming ${ }^{1}$, Xiaolin $\mathrm{Li}^{2}$, Shukai $\mathrm{Xu}^{2}$ \\ ${ }^{1}$ School of Electric Power, South China University of Technology, Guangzhou, China \\ ${ }^{2}$ Electric Power Research Institute, CSG, Guangzhou, China \\ Email: fanxinming1230@126.com
}

Received March, 2013

\begin{abstract}
Concentrated integration of large scale wind power demands stronger robustness of VSC-HVDC transmission. Based on PCHD (Port Controled Hamiltonian with Dissipation) equation, the PCHD model of voltage source converter (VSC) in abc frame and d-q rotating frame are built and the strict passivity of VSC is proved. Desired energy function is constructed and used as Lyapunov function by assigning link matrix and damping matrix. Impact from VSC equivalent dc resistance is eliminated by additional damping matrix. The IDA-PB (Interconnection and Damping Assignment Passivity-based) controller is designed based on desired equilibrium point and state variable. With different operation conditions, VSC-HVDC and its control system are simulated by software PSCAD/EMTDC, the results show the proposed control strategy has good performance and strong robustness.
\end{abstract}

Keywords: VSC-HVDC; Passivity; PCHD System; Damping Assignment; IDA-PB Control

\section{Introduction}

The use of wind power is a keystone in the policy of every country for its renewable energy development goals. Meanwhile wind farm is large-scalely developed and the capacity of a single wind farm increases to hundreds or thousands of MW [1]. HVDC with voltage source converter (VSC-HVDC) has fine dynamic characteristic and transmission flexibility, furthermore it improves the stability of power system [2,3]. In addition, VSC-HVDC is more economical for hundred megawatts wind farm connection [4]. Therefore, VSC-HVDC has obvious techno-economic advantages for the connection of large scale wind farm. The VSC-HVDC system can be operated in three modes: 1) constant dc voltage control mode; 2) constant active and reactive power control mode; and 3) constant ac voltage control mode[5]. And one of converters has to control the dc voltage to make power balanced and the dc voltage stable[6]. However four control inputs of the VSC-HVDC and their interaction make it a truly nonlinear multiple-input and multiple-output system. Furthermore the fluctuation of wind power will cause deviation of the electric variable in connection point. So VSC-HVDC need strong robustness to deliver power.

At present, voltage vector oriented double closed loop PID control[7] based on synchronously rotating frame and direct power control are commonly used. But they both have weak robustness and it is difficult to tune PID parameters. To eliminate the impacts of nonlinearity of VSC-HVDC and improve its robustness, many research works have been carried out, including feedback linearization and sliding mode control[5], adaptive back-stepping control in Ref.[8], fuzzy PI control in Ref.[9] and neural network PID in Ref. [10]. But it is still difficult to achieve ideal effects because of the multivariable structure and highly coupled nonlinearity of the VSC-HVDC system.

Passivity control is an emerging nonlinear control theory based on the energy dissipation of system[11,12]. Its profound physics meaning has significant relation with Lyapunov function. IDA-PB control is a passivity control based on PCHD. This paper, IDA-PB control strategy is proposed based on the PCHD model of VSC-HVDC. The control decreases dependence of system parameters and makes VSC-HVDC connected large wind power has smaller static error and stronger robustness. The simulation results show its advantages.

\section{Topology and Model of the VSC-HVDC}

The single line diagram of the VSC-HVDC connected a wind farm is shown in Figure 1.

The detailed structure of VSC is shown in Figure 2, the transformer reactance and power loss are equivalent to $L$ and $R$, and the parameters of the three phase circuits 
are assumed to be identical. $\boldsymbol{u}_{s a}, \boldsymbol{u}_{s b}$ and $\boldsymbol{u}_{s c}$ are phase voltage of the point of common coupling (PCC), $\boldsymbol{i}_{a}, \boldsymbol{i}_{b}$ and $\boldsymbol{i}_{c}$ are line current at PCC. $\boldsymbol{u}_{a}, \boldsymbol{u}_{b}$ and $u_{c}$ are phase voltage at the ac side of the VSC, $u_{d c}$ is the dc voltage, $\boldsymbol{i}_{d c}$ is dc current in the dc transmission line.

The mathematical model of the VSC-HVDC VSC in the three phase static frame is

$$
\left\{\begin{array}{l}
L \frac{d i_{a}}{d t}=u_{s a}-u_{a}-R i_{a}=u_{s a}-u_{d c}\left(s_{a}-\frac{1}{3} \sum_{j=a, b, c} s_{j}\right)-R i_{a} \\
L \frac{d i_{b}}{d t}=u_{s b}-u_{b}-R i_{b}=u_{s b}-u_{d c}\left(s_{b}-\frac{1}{3} \sum_{j=a, b, c} s_{j}\right)-R i_{b} \\
L \frac{d i_{c}}{d t}=u_{s c}-u_{c}-R i_{c}=u_{s c}-u_{d c}\left(s_{c}-\frac{1}{3} \sum_{j=a, b, c} s_{j}\right)-R i_{c} \\
c \frac{d u_{d c}}{d t}=S a i_{a}+S b i_{b}+s_{c} i_{c}-i_{d c}
\end{array}\right.
$$

where $S_{j}(\mathrm{j}=\mathrm{a}, \mathrm{b}, \mathrm{c})$ is the logic switch function, when $S_{j}=1$ the upper bridge arm is conductive and lower bridge arm is turn-off, when $S_{j}=0$ the contrary is the case. By Park transformation matrix and its inverse matrix, Equation (1) can be transformed to Equation (2) in d-q rotating frame

$$
\left\{\begin{array}{l}
L \frac{d i_{d}}{d t}=u_{s d}-u_{d}-R i_{d}-\omega L i_{q}=u_{s d}-s_{d} u_{d c}-R i_{d}-\omega L i_{q} \\
L \frac{d i_{q}}{d t}=u_{s q}-u_{q}-R i_{q}+\omega L i_{d}=u_{s q}-s_{q} u_{d c}-R i_{q}+\omega L i_{d} \\
\frac{2}{3} c \frac{d u_{d c}}{d t}=S_{d} i_{d}+S_{q} i_{q}-\frac{2}{3} i_{d c}
\end{array}\right.
$$

where $S_{d}$ and $S_{q}$ represent the $\mathrm{d}$ and q components of the switch function, $u_{s d}$ and $u_{s q}$ are the $\mathrm{d}$ and $\mathrm{q}$ components of the voltage at PCC, $u_{d}$ and $u_{q}$ are the $\mathrm{d}$ and q components of the voltage at ac side of VSC. The active power $P_{\mathrm{s}}$ and reactive power $Q_{\mathrm{s}}$ in the d-q rotating frame are

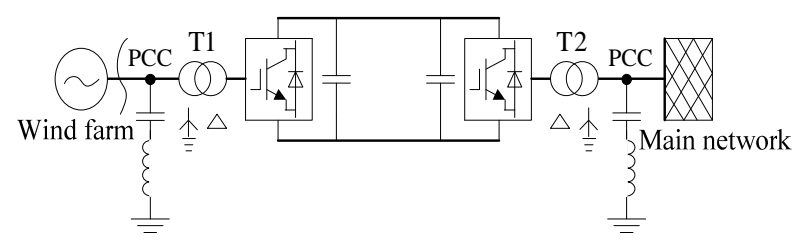

Figure 1. Wind farm VSC-HVDC connection topology.

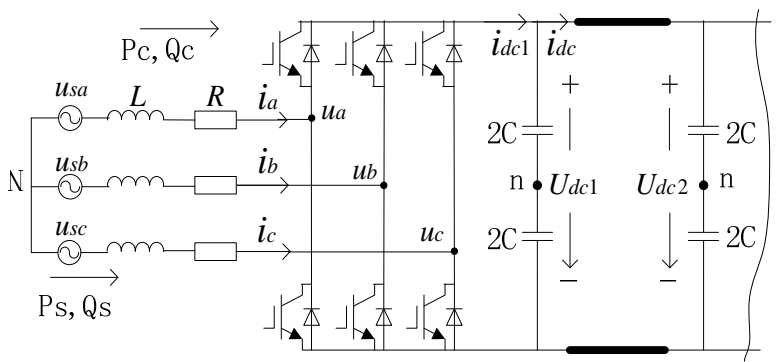

Figure 2. VSC-HVDC circuit topology.

$$
\left.\begin{array}{l}
P_{\mathrm{s}}=\frac{3}{2}\left(u_{\mathrm{sd}} i_{\mathrm{d}}+u_{\mathrm{sq}} i_{\mathrm{q}}\right) \\
Q_{\mathrm{s}}=\frac{3}{2}\left(u_{\mathrm{sd}} \dot{i}_{\mathrm{q}}-u_{\mathrm{sq}} \dot{i}_{\mathrm{d}}\right)
\end{array}\right\}
$$

When $\mathrm{d}$ axis is oriented at the vector of the voltage at PCC, then $u_{s q}=0$, and $P_{\mathrm{s}}=\frac{3}{2} u_{\mathrm{sd}} i_{\mathrm{d}}, Q_{\mathrm{s}}=\frac{3}{2} u_{\mathrm{sd}} \dot{i}_{\mathrm{q}}$, so controlling the $i_{d}$ and $i_{q}$ respectively can realize the decoupled control of $P_{\mathrm{s}}$ and $Q_{\mathrm{s}}$.

\section{PCHD Model of VSC-HVDC Converter}

Port-controlled Hamiltonian with Dissipation(PCHD) model $[13,14]$ is the form

$$
\left\{\begin{array}{l}
\dot{x}=[J(x)-\mathfrak{R}(x)] \frac{\partial H(x)}{\partial x}+g(x) u \\
y=g^{\mathrm{T}}(x) \frac{\partial H(x)}{\partial x}
\end{array}\right.
$$

where $x \in \mathrm{R}^{\mathrm{n}}$ are the state variables, $J(x)=-J^{\mathrm{T}}(x) \in \mathrm{R}^{n \times n}$ is skew-symmetric matrix, $\mathfrak{R}(x)=\mathfrak{R}^{\mathrm{T}}(x) \geq 0$ represents the dissipation, $g(x) \in \mathrm{R}^{n \times m}$ is input matrix, $H(x)$ is the system energy function, $u \in \mathrm{R}^{m}$ are input variables and $y$ are output variables. Evaluating the rate of change of the system total energy, we obtain

$$
\frac{d H(x)}{d t}=-\frac{\partial H^{\mathrm{T}}(x)}{\partial x} \mathfrak{R}(x) \frac{\partial H(x)}{\partial x}+u^{\mathrm{T}} y
$$

Because of $\mathfrak{R}(x)=\mathfrak{R}^{\mathrm{T}}(x) \geq 0$, we obtain

$$
\frac{d H(x)}{d t} \leq u^{\mathrm{T}} y
$$

Therefore energy input from external is always greater than stored in the system, this makes the system passive. Make the switch control variable $K_{i}=S_{i}-\frac{1}{3} \sum_{j=a, b, c} S_{j}$,

where $i=a, b, c$, according to Equations (1) and (4), we obtain the PCHD model of VSC in abc frame

$$
\begin{gathered}
\dot{x}=[J-\mathfrak{R}] \frac{\partial H(x)}{\partial x}+G u \\
H(x)=\frac{1}{2} x^{\mathrm{T}} M x
\end{gathered}
$$

where $x=\left[\begin{array}{llll}i_{a} & i_{b} & i_{c} & u_{d c}\end{array}\right]^{\mathrm{T}}, \quad u=\left[\begin{array}{llll}u_{s a} & u_{s b} & u_{s c} & 0\end{array}\right]^{\mathrm{T}}$

$$
\begin{aligned}
J & =\left[\begin{array}{cccc}
0 & 0 & 0 & -K_{a} / L C \\
0 & 0 & 0 & -K_{b} / L C \\
0 & 0 & 0 & -K_{c} / L C \\
K_{a} / L C & K_{b} / L C & K_{c} / L C & 0
\end{array}\right] \\
R & =\left[\begin{array}{cccc}
R / L^{2} & 0 & 0 & 0 \\
0 & R / L^{2} & 0 & 0 \\
0 & 0 & R / L^{2} & 0 \\
0 & 0 & 0 & 1 /\left(R_{\mathrm{dc}} C^{2}\right)
\end{array}\right]
\end{aligned}
$$




$$
G=\left[\begin{array}{cccc}
1 / L & 0 & 0 & 0 \\
0 & 1 / L & 0 & 0 \\
0 & 0 & 1 / L & 0 \\
0 & 0 & 0 & 1 / C
\end{array}\right], \quad M=\left[\begin{array}{cccc}
L & 0 & 0 & 0 \\
0 & L & 0 & 0 \\
0 & 0 & L & 0 \\
0 & 0 & 0 & C
\end{array}\right]
$$

and $R_{\mathrm{dc}}=u_{\mathrm{dc}} / i_{\mathrm{dc}}$ i.e. the equivilent resistor at the dc side of VSC. By Park transformation, Equation (7) can be transformed to PCHD model in d-q synchronously rotating frame as Equation (9)

$$
\dot{x}=[J-\mathfrak{R}] \frac{\partial H(x)}{\partial x}+G_{1} u+G_{2} \dot{i}_{d c}
$$

where $x=\left[\begin{array}{lll}L i_{d} & L i_{q} & C u_{d c}\end{array}\right]^{\mathrm{T}}, G_{2}=\left[\begin{array}{lll}0 & 0 & -1\end{array}\right]^{\mathrm{T}}$

$$
\begin{aligned}
J & =\left[\begin{array}{ccc}
0 & -\omega L & -\frac{3}{2} S_{d} \\
\omega L & 0 & -\frac{3}{2} S_{q} \\
\frac{3}{2} S_{d} & \frac{3}{2} S_{q} & 0
\end{array}\right], \mathfrak{R}=\left[\begin{array}{ccc}
R & 0 & 0 \\
0 & R & 0 \\
0 & 0 & 0
\end{array}\right], \\
G_{1} & =\left[\begin{array}{lll}
1 & 0 & 0 \\
0 & 1 & 0 \\
0 & 0 & 0
\end{array}\right], \quad u=\left[\begin{array}{lll}
u_{s d} & u_{s q} & 0
\end{array}\right]^{\mathrm{T}} .
\end{aligned}
$$

The energy function of the system is defined as

$$
H(x)=\frac{1}{2 L} x_{1}^{2}+\frac{1}{2 L} x_{2}^{2}+\frac{1}{3 C} x_{3}^{2}
$$

\section{IDA-PB Controller Design of Converter}

According to IDA-PB control theory[14][15], it need to find out functions $\beta(x), J_{\mathrm{a}}(x), \Re_{\mathrm{a}}(x)$ and vector function $K(x)$ for the desired stable equilibrium points $x^{*} \in \mathrm{R}^{\mathrm{n}}$ by assigning interconnection and damping marix and make they meet the Equation (11)

$$
\begin{aligned}
& \left\{J[x, \beta(x)]+J_{\mathrm{a}}(x)-\left[\mathfrak{R}(x)+\Re_{\mathrm{a}}(x)\right]\right\} K(x) \\
= & -\left[J_{\mathrm{a}}(x)-\mathfrak{R}_{\mathrm{a}}(x)\right] \frac{\partial H(x)}{\partial x}+g[x, \beta(x)]
\end{aligned}
$$

They meets conditions as follows

$$
\begin{array}{r}
J_{\mathrm{d}}(x)=J[x, \beta(x)]+J_{\mathrm{a}}(x)=-\left\{J[x, \beta(x)]+J_{\mathrm{a}}(x)\right\}^{\mathrm{T}} \\
\Re_{\mathrm{d}}(x)=\Re(x)+\Re_{\mathrm{a}}(x)=\left[\Re(x)+\Re_{\mathrm{a}}(x)\right]^{\mathrm{T}} \geq 0 \\
\frac{\partial K(x)}{\partial x}=\left(\frac{\partial K(x)}{\partial x}\right)^{\mathrm{T}}, K\left(x^{*}\right)=-\left.\frac{\partial H(x)}{\partial x}\right|_{x=x^{*}}
\end{array}
$$

The dc voltage is expect to be the reference value of $u_{d c}{ }^{*}, i_{d}$ and $i_{q}$ are determined by the requirements of decoupled control of active power and reactive power. Therefore, the anticipated stable equilibrium points are $x_{1}^{*}=\operatorname{Lid}^{*}, x_{2}^{*}=\operatorname{Li}_{q}^{*}, x_{3}^{*}=C u_{d c}{ }^{*}$. The PCHD model Equation (9) can be written as the Equation (15)

$$
\dot{x}=\left[J-\Re^{\prime}\right] \frac{\partial H(x)}{\partial x}+G_{1} u
$$

where $\mathfrak{R}^{\prime}=\left[\begin{array}{ccc}R & 0 & 0 \\ 0 & R & 0 \\ 0 & 0 & 3 /\left(2 R_{\mathrm{dc}}\right)\end{array}\right]$.
So dc equivalent resistor exist in converter dissipation matrix. Its impacts are to be eliminated by assigning damping matrix. Make that

$$
J_{\mathrm{a}}(x)=0, \quad \mathfrak{R}_{\mathrm{a}}=\left[\begin{array}{ccc}
0 & 0 & 0 \\
0 & 0 & 0 \\
0 & 0 & 3 /\left(2 R_{\mathrm{dc}}\right)
\end{array}\right]
$$

According to Equations (11), (12) and (13), we obtain Equation (16)

$$
\left[J_{\mathrm{d}}(x)-\mathfrak{R}_{\mathrm{d}}(x)\right] K(x)=\mathfrak{R}_{\mathrm{a}}(x) \frac{\partial H(x)}{\partial x}+G_{1} u
$$

Then the expasion of Equation (16) is Equation (17)

$$
\left\{\begin{array}{l}
S_{\mathrm{d}}=-\frac{2}{3}\left[\left(\omega L K_{2}+R K_{1}+u_{s d}\right) / K_{3}\right] \\
S_{\mathrm{q}}=-\frac{2}{3}\left[\left(-\omega L K_{1}+R K_{2}+u_{s q}\right) / K_{3}\right]
\end{array}\right.
$$

Setting $K_{1}=k_{1}\left(x_{1}\right), K_{2}=k_{2}\left(x_{2}\right)$ and $K_{3}=k_{3}\left(x_{3}\right)$, according to Equation (14), we obtain

$$
\left\{\begin{array}{l}
k_{1}\left(x_{1}^{*}\right)=-x_{1}^{*} / L=-i_{d}{ }^{*} \\
k_{2}\left(x_{2}^{*}\right)=-x_{2}^{*} / L=-i_{q}{ }^{*} \\
k_{3}\left(x_{3}^{*}\right)=-2 x_{3}^{*} / 3 C=-2 u_{\mathrm{dc}}{ }^{*} / 3
\end{array}\right.
$$

Based on above conditions, a set of solution for $K_{1}, K_{2}$ and $K_{3}$ is

$$
\left\{\begin{array}{l}
k_{1}\left(x_{1}\right)=-x_{1}^{*} / L+\alpha\left(x_{1}-x_{1}^{*}\right) \\
k_{2}\left(x_{2}\right)=-x_{2}^{*} / L+\beta\left(x_{2}-x_{2}^{*}\right) \\
k_{3}\left(x_{3}\right)=-2 x_{3}^{*} / 3 C+\gamma\left(x_{3}-x_{3}^{*}\right)
\end{array}\right.
$$

where $\alpha, \beta, \gamma>0$.

Substituting Eqation (19) to Eqation (17) and considering that the dc voltage of VSC is equal to desired stable equilibrium voltage i.e. $U_{d c}=u_{d c}^{*}$ at steady state, then the VSC control laws can be obtained and simplified as

$$
\begin{aligned}
u_{d} & =u_{s d}-\omega L i_{q}^{*}+\alpha R L\left(i_{d}-i_{d}^{*}\right) \\
& -R i_{d}^{*}+\beta \omega L^{2}\left(i_{q}-i_{q}^{*}\right) \\
u_{q} & =u_{s q}+\omega L i_{d}^{*}+\beta R L\left(i_{q}-i_{q}^{*}\right) \\
& -R i_{q}^{*}-\alpha \omega L^{2}\left(i_{d}-i_{d}^{*}\right)
\end{aligned}
$$

where $\dot{i}_{d}{ }^{*}$ and $\dot{i}_{q}{ }^{*}$ can be obtained by outer-loop control.

In order to facilitate active power setting and voltage control for wind farm, active power and constant voltage control are used at the outer-loop controller of sending end, and constant dc voltage and constant voltage controls are used at receiving end, Figure 3 shows the control diagram.

\section{Simulation and Analysis}

The VSC-HVDC linked wind farm and its control strategy are modeled and simulated by software PSCAD/ 
EMTDC. The rated dc voltage of the VSC-HVDC is $\pm 160 \mathrm{kV}$ and the base capacity is $100 \mathrm{MVA}$. The power production of the wind farm is $180 \mathrm{MW}$, the PCC voltage of both ends is $110 \mathrm{kV}$. The simulation time span is $20 \mathrm{~s}$. Comparison is made between PI double closed-loop control and IDA-PB control, and the outer-loop PI parameters of both are identical so as to ensure comparison valid. Figure 4 to Figure 7 show the steady simulation results.

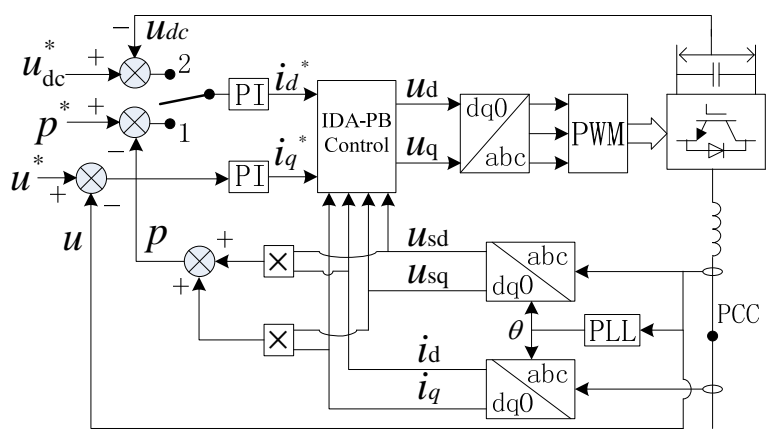

Figure 3. IDA-PB control strategy for both ends VSC.

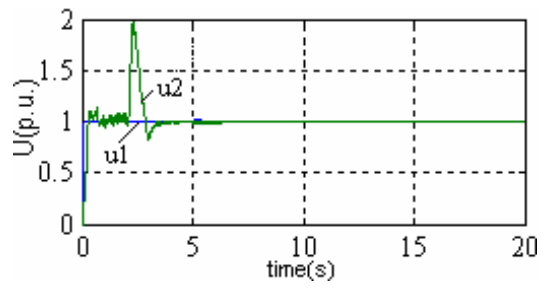

(a) Voltage pu value in PCC under PI double closed-loop control

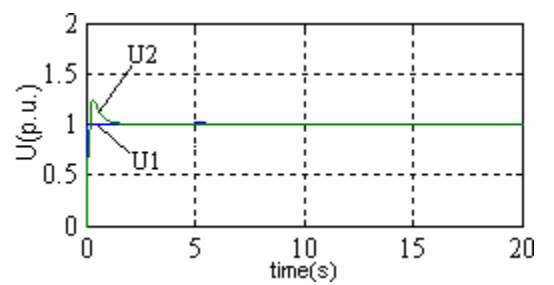

(b) Voltage pu value in PCC under IDA-PB control

Figure 4. Voltage value in PCC under two control strategies.

Simulation resluts in Figure 4(b), Figure 5(b), Figure 6 and Figure 7(b) show ac voltage, wind farm power production, active power transmitted by VSC-HVDC, dc voltage and direct current reach set value quickly and keep stable, no oscillation and very small overshoot under IDA-PB control. Comparison demonstrates that IDA$\mathrm{PB}$ control make the system has stronger robustness.

In order to compare dynamic response performances, make the wind speed have a step change at $10 \mathrm{~s}$ and $15 \mathrm{~s}$ under both control modes, as shown in Figure 8. Figure 9 shows the simulation results of each variable.

Figure 9(a) shows the dc voltage at sending end has little fluctuation and smaller flutter under IDA-PB control. The receiving end dc voltage is stabilized on set value and error is $\pm 0.05 \mathrm{kV}$ under IDA-PB control, but it fluctuates greatly and need much time to reach stability under PI double closed-loop control, as shown in Figure 9(b).

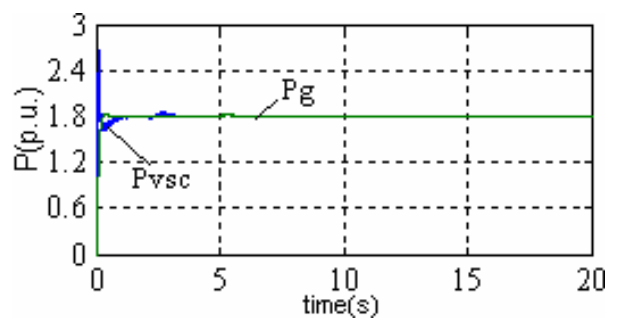

(a) Wind power and transmited powoer under PI double closed-loop control.

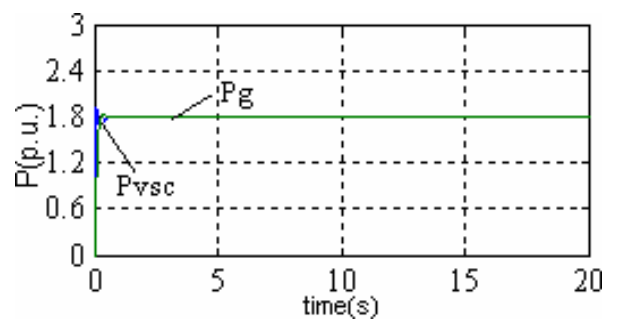

(b) Wind power and transmited powoer under IDA-PB control

Figure 5. Wind power and transmited power under two control strategies.

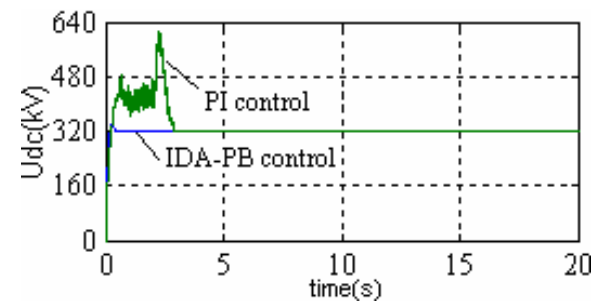

Figure 6. Direct voltage of receiving end under two control strategies.

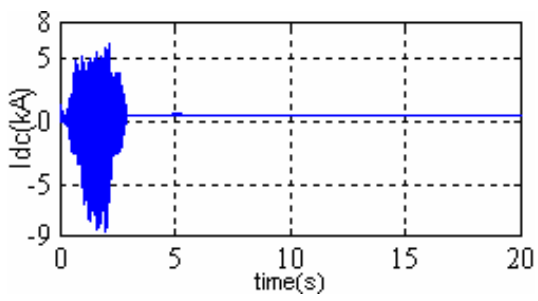

(a) Direct current under PI double closed-loop control

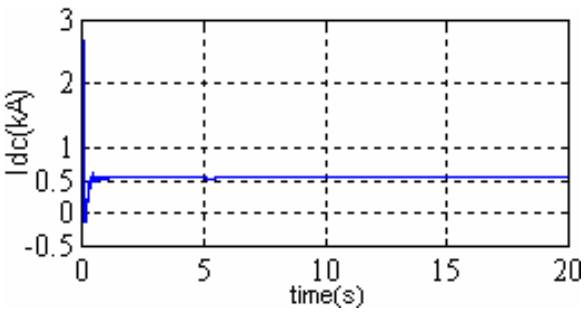

(b) Direct current under IDA-PB control

Figure 7. Direct current under two control strategies. 


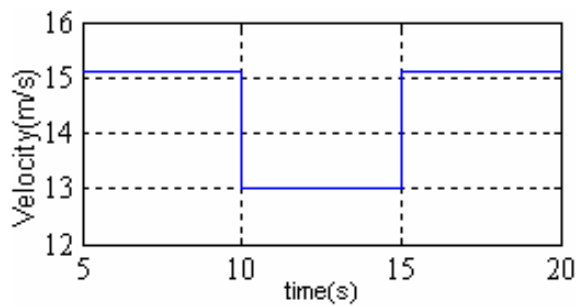

Figure 8. Wind speed.

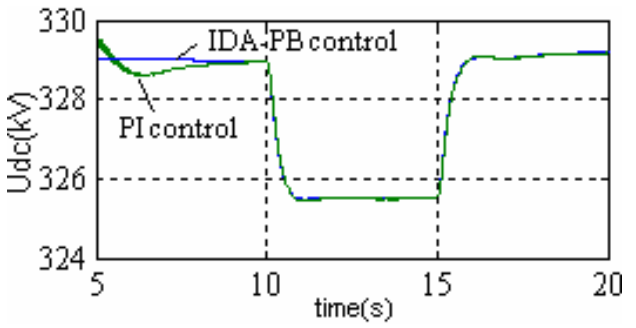

(a) Direct voltage of sending end under two control strategies

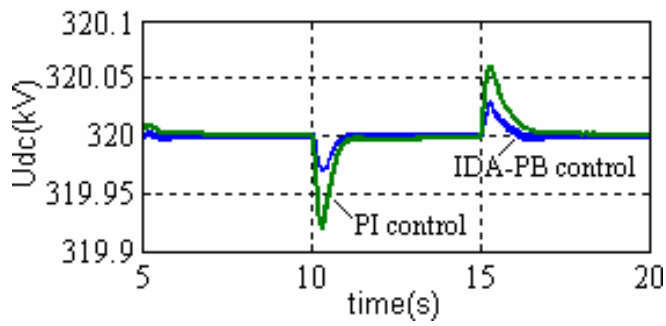

(b) Direct voltage of receiving end under two control strategies

Figure 9. Direct voltage under step wind disturbance.

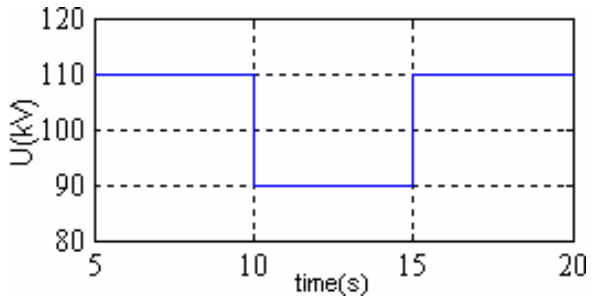

Figure 10. Voltage step change at PCC of receiving end.

Setting the voltage amplitude step change at the PCC of receiving end to simulates voltage disturbance of connected grid, as shown in Figure 10. Figure 11 and Figure 12 show the results.

Figure 11 shows the ac voltage has little ripple and better steady and dynamic performance under IDA-PB control when the voltage at PCC of receiving end changes. And dc voltage at receiving end has smaller static error, it recover to set value quickly after disturbance under IDA-PB control, as shown in Figure 12.

\section{Conclusions}

The robustness of the VSC-HVDC transmission linked large wind power is discussed, a IDA-PB control strategy is proposed for converter in this paper. The proposed

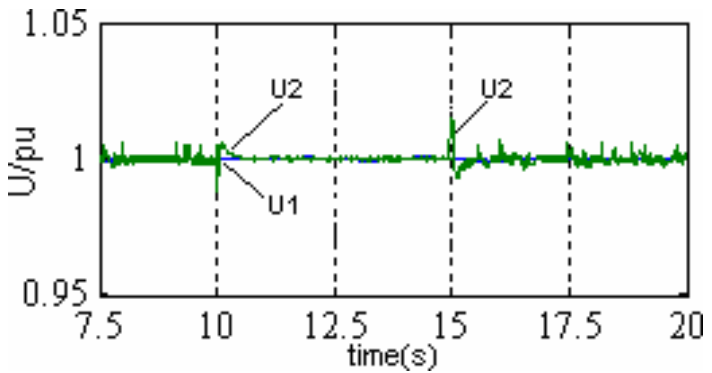

(a) Voltage pu value in PCC under PI double closed-loop control

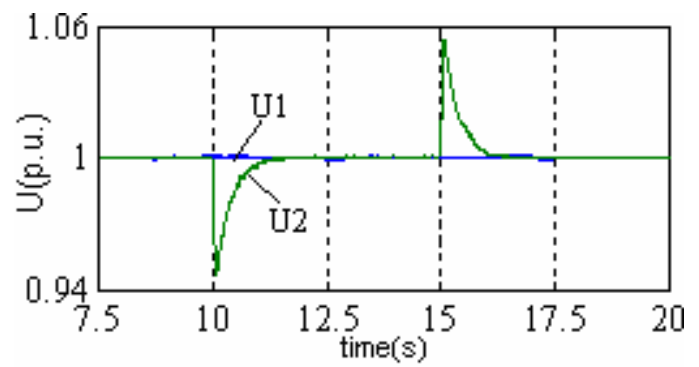

(b) Voltage pu value in PCC under IDA-PB control

Figure 11. Voltage value in PCC under step voltage disturbance at PCC of receiving end.

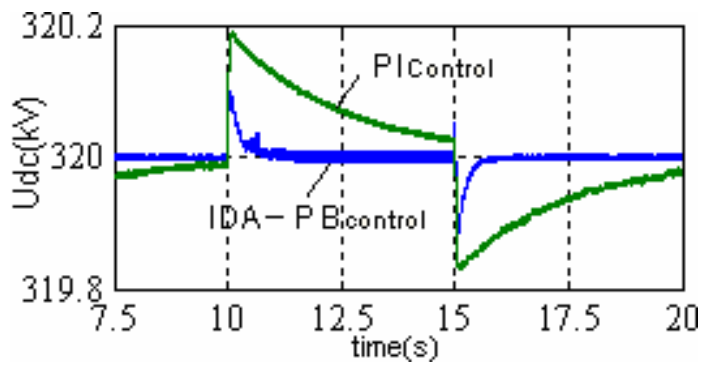

Figure 12. Direct voltage of receiving end under step voltage disturbance at PCC of receiving end.

control strategy has merits as follows:

1) The system energy function has passivity characteristic. It is accord with Lyaapunov stability theory and has clear physical meaning, and ideal controller can be obtained by making best of it.

2) PCHD function has general affine nonlinear structure. Its essence is a nonlinear control strategy and it is apt to nonlinear characteristic of VSC-HVDC.

3) IDA-PB control simplified controller design of PCHD model. Damping assignment decrease the impact from system parameter deviation and make the energy function non-growth and minimum value at equilibrium point.

4) The proposed strategy achieved decoupled control of four inputs. And the control law is easy and of practical value for engineering application.

\section{Acknowledgements}

This work was supported by the national high technology 
research and development program of China (863 Program) (No. 2011AA05A102).

\section{REFERENCES}

[1] L. Y. Zhang, T. L. Ye, Y. Z. Xin, et al, "Problems and Measures of Power Grid Accommodating Large Scale Wind Power," Proceedings of the CSEE, Vol. 30, No. 25, 2010, pp. 1-9.

[2] N. Flourentzou, V. G. Agelidis and G. D. Demetriades, "VSC-based HVDC Power Transmission Systems: An Overview," IEEE Transactions on Power Electronics, Vol. 24, No. 3, 2009, pp. 594-599. doi:10.1109/TPEL.2008.2008441

[3] S. M. Muyeen, R. Takahashi and J. Tamura, "Operation and Control of HVDC-connected Offshore Wind Farm," IEEE Transactions on Sustainable Energy, Vol. 1, No. 1, 2010, pp. 30-37. doi:10.1109/TSTE.2010.2041561

[4] P. Bresesti, W. L. Kling and R. L. Hendriks, "HVDC Connection of Offshore Wind Farms to the Transmission System," IEEE Transactions on Energy Conversion, Vol. 22, No. 1, 2007, pp. 37-43. doi:10.1109/TEC.2006.889624

[5] A. Moharana and P. K. Dash, "Input-output Linearization and Robust Sliding-mode Controller for the VSC-HVDC Transmission Link," IEEE Transactions on Power Delivery, Vol. 25, No. 3, 2010, pp. 1952-1961. doi:10.1109/TPWRD.2010.2042469

[6] L. D. Zhang, L. Harnefors and H. P. Nee, "Interconnection of Two Very Weak AC Systems by VSC-HVDC Links Using Power Synchronization Control,” IEEE Transactions on Power Systems, Vol. 26, No. 2, 2011, pp. 345-347.

[7] M. Y. Guan and Z. Xu, "Modeling and Control of Modular Multilevel Converter in HVDC Transmission," Automation of Electric Power Systems, Vol. 34, No. 19, 2010, pp. 64-68.

[8] S. Y. Ruan, G. J. Li, X. H. Jiao, et al., “Adaptive Control Design for VSC-HVDC Systems Based on Backstepping Method," Electric Power Systems Research, Vol. 77, No. 6, 2007, pp. 559-565. doi:10.1016/j.epsr.2006.05.006

[9] S. H. Li, T. A. Haskew and L. Xu, "Control of HVDC Light System Using Conventional and Direct Current Vector Control Approaches," IEEE Transactions on Power Electronics, Vol. 25, No. 12, 2010, pp. 3106-3118. doi:10.1109/TPEL.2010.2087363

[10] S. Li, Z. X. Wang, G. Q. Wang, et al., "PID Neural Network Sliding-mode Controller for Three-level Offshore Wind Power VSC-HVDC Converter," Proceedings of the CSEE, Vol. 32, No. 4, 2012, pp. 20-28.

[11] R. Ortega and G. C. Eloísa, "Interconnection and Damping Assignment Passivity-based Control: A Survey," European Journal of Control, Vol. 10, No. 5, 2004, pp. 1-27. doi:10.3166/ejc.10.432-450

[12] T. S. Lee, "Lagrangian Modeling and Passivity Based Control of Three-phase AC/DC Voltage-source Converters," IEEE Transaction on Industrial Electronics, Vol. 51, No. 4, 2004, pp. 892-902. doi:10.1109/TIE.2004.831753

[13] J. H. Wang, L. P. Huang and X. Y. Yang, "Power Control of Three-phase Boost-type PWM Rectifier Based on Passivity," Proceedings of the CSEE, Vol. 28, No. 21, 2008, pp. 20-25.

[14] R. Ortega, A. van der Schaft, B. Maschke, et al., "Interconnection and Damping Assignment Passivity-based Control of Port-controlled Hamiltonian System," Automatica, Vol. 38, No. 4, 2002, pp. 585-596. doi:10.1016/S0005-1098(01)00278-3

[15] R. Ortega, A. van der Schaft, F. Castaños, et al., "Control by Interconnection and Standard Passivity-based Control of Port-hamiltonian Systems," IEEE Transactions on $\mathrm{Au}$ tomatic Control, Vol. 53, No. 4, 2008, pp. 2527-2542. doi:10.1109/TAC.2008.2006930 